Small-scale patterning methods for digital image correlation under scanning electron microscopy

This article has been downloaded from IOPscience. Please scroll down to see the full text article.

2011 Meas. Sci. Technol. 22125501

(http://iopscience.iop.org/0957-0233/22/12/125501)

View the table of contents for this issue, or go to the journal homepage for more

Download details:

IP Address: 141.211.173.82

The article was downloaded on 06/04/2012 at 15:58

Please note that terms and conditions apply. 


\title{
Small-scale patterning methods for digital image correlation under scanning electron microscopy
}

\author{
A D Kammers and S Daly \\ The University of Michigan, Department of Mechanical Engineering, 2350 Hayward St, Ann Arbor, \\ MI 48109, USA \\ E-mail: akammers@umich.edu
}

Received 27 June 2011, in final form 20 September 2011

Published 19 October 2011

Online at stacks.iop.org/MST/22/125501

\begin{abstract}
Digital image correlation (DIC) is a powerful, length-scale-independent methodology for examining full-field surface deformations. Recently, it has become possible to combine DIC with scanning electron microscopy (SEM), enabling the investigation of small-scale deformation mechanisms such as the strains accommodated within grains in polycrystalline metals, or around micro-scale constituents in composite materials. However, there exist significant challenges that need to be surmounted before the combination of DIC and SEM (here termed SEM-DIC) can be fully exploited. One of the primary challenges is the ability to pattern specimens at microstructural length scales with a random, isotropic and high contrast pattern needed for DIC. This paper provides a thorough survey of small-scale patterning methods for SEM-DIC and discusses their advantages and disadvantages for different applications.
\end{abstract}

Keywords: digital image correlation, surface patterning, SEM-DIC, nanoparticles, electron beam lithography, microstructure, deformation mechanisms

(Some figures in this article are in colour only in the electronic version)

\section{Introduction}

Understanding the effect of microstructure on the macroscopic response of materials requires new experimental techniques to track full-field deformations on small length scales with nano-scale resolution. To fulfill this need, the digital image correlation (DIC) technique has recently been combined with scanning electron microscopy (SEM) [1-3]. This methodology (here termed SEM-DIC) allows investigators to link the microstructural features of a material to its macro-scale material properties. This approach enables the acquisition of a substantial amount of new information, including, for example, the effect of the Schmid factor of a grain and the distance from a grain boundary on local stress concentrations [4], or the characterization of the microstructural strain distribution at the interface of ferritic and martensitic regions in dual-phase steel [5].
Digital image correlation (DIC) is a non-contact optical technique used to measure displacements on the surface of an object, from which Lagrangian strains can be calculated. A brief overview of the process will be provided later in this section (for detailed information on the DIC technique see $[6,7]$ and the references contained therein). The SEMDIC technique introduces certain challenges that are not encountered in macro-scale DIC experiments. One such challenge is the need to pattern the surface with the smallscale tracking markers. DIC requires the sample surface to have an isotropic, high contrast and random surface pattern. Creating this pattern is relatively straightforward at millimeter and larger fields of view, by using airbrushing or toner powder. However, these methods do not work at the micron fields of view used in SEM, and new approaches to create suitable tracking patterns for DIC at these smaller length scales are needed. The second primary challenge in the ongoing development of SEM-DIC is the accurate calculation of small 


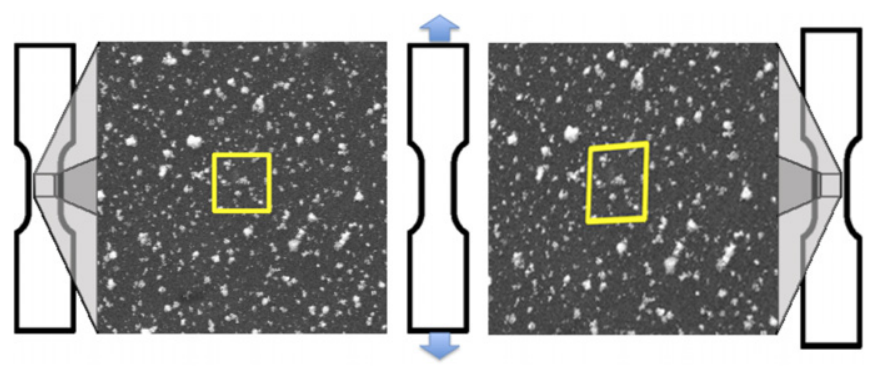

Figure 1. Illustration of a DIC subset used to track grayscale value as a test sample is deformed. The left image shows the reference (undeformed) sample and the right image shows the deformed sample. The correlation algorithm tracks the grayscale value within subsets to determine surface displacements. Note that the subset box is approximately $3 \times 3$ speckles in height and width, which is a good 'rule of thumb' to follow [6].

motions in the microstructure, such as the motion of two grains with respect to each other, from electron micrographs. This challenge, including the effect of stress relaxation and a method for calculating relative displacements, will be discussed in an upcoming paper. The SEM image scanning process is time consuming and errors in the positioning of the electron beam introduce artificial displacements into the images. The future publication will discuss improvements to current SEM distortion correction techniques [1] and provide additional steps to ensure distortion-free data. Additional challenges faced with SEM-DIC include limitations on the size of the test samples that can be investigated and the size of the straining stage that can fit inside of the SEM chamber. Sample charging can also be an issue with nonconducting samples. The SEM increases image noise over that seen with optical imaging. Finally, SEM usage and patterning methods can be expensive.

The DIC technique was developed by researchers at the University of South Carolina in the 1980s [8, 9]. The surface of a DIC test sample must have an isotropic, high contrast and random pattern. To calculate full-field displacements, the surface pattern is analyzed in subsets as shown in figure 1, where each subset contains a unique grayscale value resulting from the pattern. Displacements, and thus Lagrangian strains, are calculated by comparing the deformed images to an initial reference image, and determining how a reference image subset needs to be distorted to match the grayscale distribution found in the deformed images. Two-dimensional DIC uses one image sensor and is suitable for applications that are not subject to out-of-plane motions. Three-dimensional DIC uses two image sensors to track surface displacements in three dimensions. DIC is a flexible and powerful tool that can be used across a wide range of time and length scales. It can be applied to both high-speed impact [10-13] and low strainrate experiments [14-20]. The DIC technique has no inherent length scale, and thus is applicable to experiments covering a broad range of fields of view (FOVs) from civil structures [21] to the micro- and nano-scale [4, 5, 20, 22, 23].

The use of SEM for DIC image capture requires new small-scale patterning methods on the length scale of the microstructure. The appropriate speckle size follows a general rule that each speckle should cover approximately $3 \times 3$ pixels and each subset should contain at least $3 \times 3$ speckles [6]. Following this general rule, a $100 \mu \mathrm{m}$ horizontal field width $(\mathrm{HFW})$ captured by a SEM with a $1024 \times 884$ pixel resolution requires an average speckle diameter of $300 \mathrm{~nm}$. This speckle size is well below the capabilities of patterning methods commonly used for DIC at larger scales, such as airbrushing and using toner powder [24]. Creating a nano-scale random isotropic pattern is difficult. SEM-DIC patterning methods that have been previously investigated by others include nanoparticle (NP) techniques [25], chemical vapor thin film rearrangement [26] and patterning of polymeric substrates through the use of a contact lithography method [27]. Patterning techniques have continued to evolve with recent additions including through thin film ablation [4], photolithography [24, 26] and electron-beam lithography [28].

There have been a number of other recent developments important to small-scale DIC analyses. Image stitching has been used with DIC to obtain results over a large FOV while still maintaining a high spatial resolution [29]. In metallic materials, the stitched FOV allows for more grains to be investigated without losing the spatial resolution achieved with a smaller FOV. Another noteworthy recent development to SEM-DIC is the successful use of 3D DIC within the SEM chamber in 2011 by Zhu et al [30]. This is achieved by capturing duplicate images of the sample surface after tilting the SEM stage, and modifying distortion removal techniques developed for 2D SEM-DIC to quantify out-of-plane motion and tilt. Accuracy of the positioning stage is important for 3D SEM-DIC, as inaccuracies in tilt can introduce artificial strains.

Combining the electron backscatter diffraction (EBSD) and SEM-DIC techniques is critical to establishing a relationship between localized strain and microstructure. EBSD is a widespread technique used in microstructural characterization to determine grain structure, grain orientation, texture and phases. Briefly, for an EBSD analysis, the surface of the sample is polished to remove any residual surface damage. The sample does not need to be electron translucent and only needs to be small enough to fit inside the SEM chamber, making sample preparation significantly easier than that required for electron diffraction in a transmission electron microscope (TEM). The sample is tilted approximately $20^{\circ}$ in relation to the electron beam, and the beam is rastered across a selected area of the surface. As the e-beam scans across the surface, electrons are diffracted by the crystal lattice. These backscattered electrons impinge on a phosphor screen near the tilted sample and create a diffraction pattern appearing as Kikuchi bands [31], which are indexed by EBSD software to determine the orientation of the crystal at that location. The software uses the crystal orientation to produce an orientation imaging microscopy (OIM) micrograph showing grain size, shape and misorientation as well as information on texture, phase distribution and strain state. EBSD is a highspeed process, capable of indexing 400 points per second with a spatial resolution of $20 \mathrm{~nm}$ for copper using a field emission gun (FEG) [31]. In this work, using FEG-SEM to analyze pure $\mathrm{Al}$, an average speed of 50 points per second 
is achieved. Details on the EBSD technique can be found in many references (for examples see [31, 32]). DIC experiments combined with EBSD are not without challenges, but have proven to be a valuable means for comparing microstructural deformations directly to the underlying crystallography.

This paper presents patterning techniques that cover a wide range of FOVs and includes inexpensive and quick NP patterning as well as precise but costly electronbeam lithography. New observations of the preferred NP arrangement following EBSD are also presented. In addition to patterning, a focused ion beam (FIB) technique to apply DIC and EBSD FOV alignment markers, and the steps necessary to align the two fields are covered. This is the first paper in a two-part paper series detailing the steps necessary to combine SEM-DIC with EBSD to relate deformation and strain to the microstructure of the material being studied. The upcoming second paper will cover SEM distortion corrections and a technique to track small-magnitude relative motion at the scale of the microstructure, such as motion of one grain in relation to another grain. It will conclude with an in situ tensile test on 6061-T6 Al to display the results that can be achieved by combining the SEM-DIC approach with EBSD mapping.

\section{Results and discussion}

\subsection{Patterning for EBSD area designation and alignment with DIC-calculated strain fields}

A key advantage of SEM-DIC is the ability to directly compare full-field, in situ deformations to the material microstructure. The first step in linking the strain field and microstructure is polishing the surface to a mirror finish. All the techniques applied in this paper have been developed for representative materials of $99.99 \%$ pure $\mathrm{Al}$ and 6061-T6 Al, which were both prepared for EBSD by the following method. The samples were first polished with 400, 600 and 800 grit $\mathrm{SiC}$ papers with deionized (DI) water. Buehler MetaDi supreme polycrystalline diamond suspensions were then used, starting at a grit size of $9 \mu \mathrm{m}$, followed by 3 and $1 \mu \mathrm{m}$. The final polishing step required polishing with a soft, neoprene-style Mager Scientific Dura Chem PC-740 cloth and Buehler MasterMet colloidal silica suspension 40-6370. After achieving a mirror surface finish, the area of interest was designated using FIB deposition in order to match up the DIC FOV with the pre-test EBSD, and to determine the post-mortem microstructure after thermo-mechanical loading.

\subsubsection{Area of interest designation. It is critical that the FOV} observed during tensile testing is aligned with the FOV on which the pre-test OIM microstructure map has been collected. In this investigation, this alignment is complicated by the fact that tensile testing and EBSD analysis occur in two different SEMs. This is not an uncommon occurrence, due to equipment limitations where tensile stages are compatible with a nonEBSD equipped microscope (as in the authors' case), or where different research facilities must be used for in situ testing and EBSD analysis. In the case of separate SEMs for EBSD and testing, markers are applied to the surface of the test sample

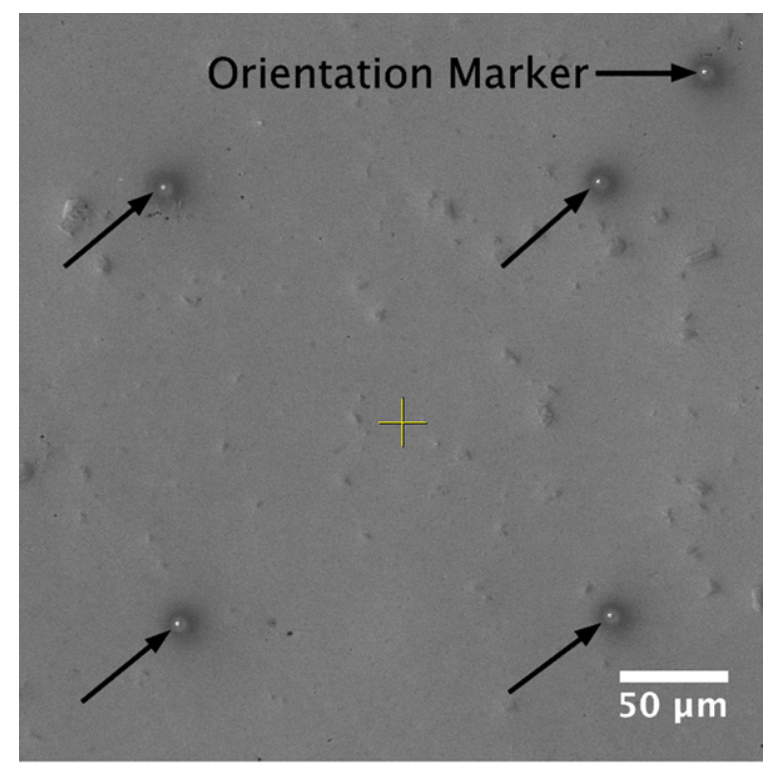

Figure 2. $1 \mu \mathrm{m}$ diameter Pt markers applied to the surface of a 6061-T6 Al test sample to denote the DIC area of interest. The four central markers in combination with the top-right orientation marker allow for alignment of the DIC and EBSD fields of view.

prior to the initial EBSD analysis for later alignment of the testing and EBSD areas. In this investigation, markers were applied using a FEI Quanta 200 3D SEM equipped with a FIB and gas injection system (GIS). The GIS was used to deposit platinum $(\mathrm{Pt})$ markers at the corners of the desired FOV, as shown in figure 2. One additional marker was placed outside of the field of view to denote test sample orientation.

The Pt markers in figure 2 are $1 \mu \mathrm{m}$ in diameter, and can be easily found at the $1100 \times$ magnification used for the test. At higher magnifications, the marker diameter can be scaled down to as small as $100 \mathrm{~nm}$ in diameter [33]. The rings surrounding the Pt markers visible in figure 2 result from two phenomena. First, only a partial amount of the Pt gas interacts with the ion beam. The amount of the Pt gas that avoids the ion beam impacts the surface and deposits itself. Second, the ion beam scatters as it approaches the surface and deposits $\mathrm{Pt}$ outside of the desired area [34]. The halo surrounding the Pt marker scales with the size of the marker and can be reduced by using smaller Pt markers as shown in figure 3. Equivalentsized markers deposited by the electron beam also possess this Pt halo.

The Pt markers adhere well to the surface of test samples and are capable of withstanding light polishing with colloidal silica as well as ultrasonic cleaning. In a paper by Carroll et al [29], $25 \mu \mathrm{m}$ wide Vickers microindentations are used instead of Pt markers to designate the FOV. These indentations have the advantage over Pt markers of being more permanent and having sharp corners to assist with alignment, although similar aligning features could be applied with the FIB. The main disadvantages of Vickers markers are their large size and the residual stress induced in the test sample near the markers. Additionally, microindentations would not be applicable to thin foil samples, whereas FIB-deposited Pt markers have been successfully applied by the authors to $2 \mu \mathrm{m}$ thick metallic foils. 


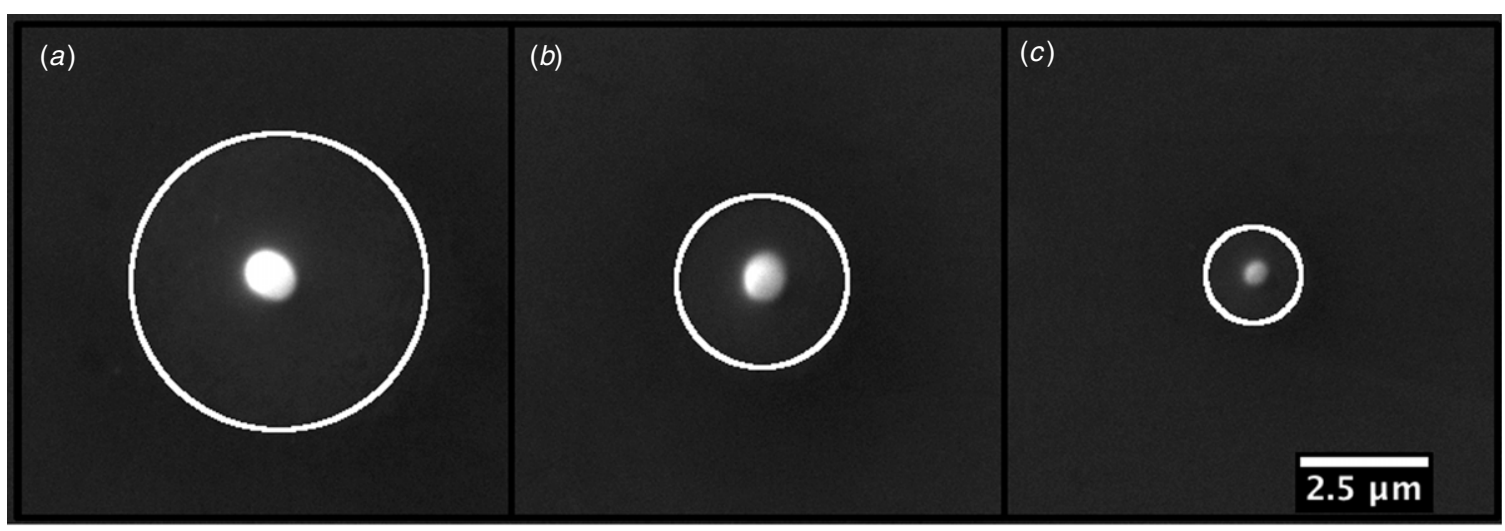

Figure 3. Pt markers deposited on the surface of a pure Al sample. The Pt halo surrounding the markers scales with the marker size. The markers are $1 \mu \mathrm{m}$ in diameter $(a), 800 \mathrm{~nm}$ in diameter $(b)$ and $400 \mathrm{~nm}$ in diameter $(c)$.

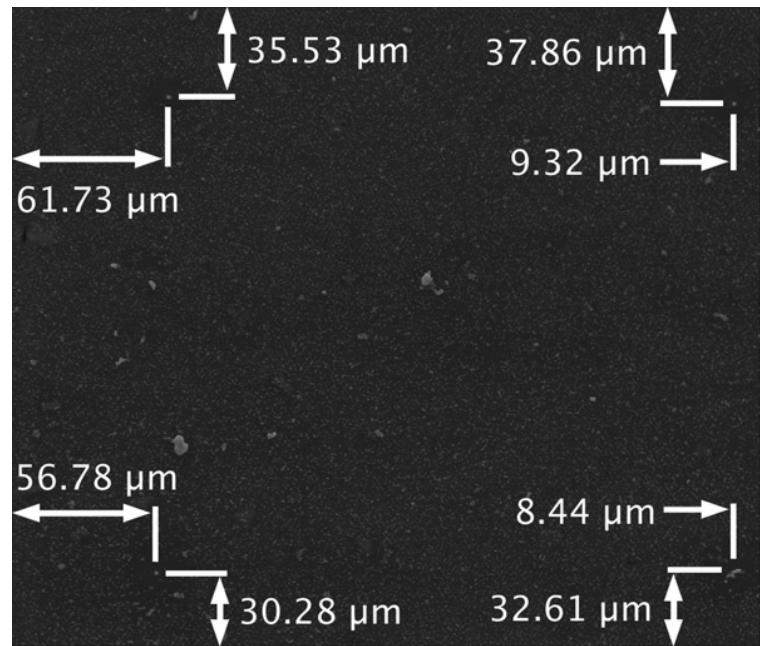

Figure 4. FOV of a final unloaded test image. The dimensions recording the locations of the Pt markers are used to position the EBSD area. The orientation marker is outside of the FOV, but its position is also recorded prior to transferring the test sample to the EBSD SEM

\subsubsection{EBSD area alignment. Following the application of} Pt markers to designate the area of interest, a pre-test EBSD analysis of the sample surface was performed. The alignment of the pre-test EBSD area with the initial test FOV markers was straightforward, only requiring the area of interest designated by the Pt markers to be centered in the EBSD area. Note that after the sample undergoes thermo-mechanical loading and returns to the unloaded state, the markers will no longer be equally spaced from each corner of the FOV, as shown in figure 2. In the final test image, the location of each marker in relation to the nearest edges was recorded as shown in figure 4. Upon loading the sample into the EBSD-equipped SEM, the sample was rotated until the dimensions of the markers within the EBSD area matched those recorded for the final test image FOV. This ensured that the EBSD FOV was aligned with the final test image FOV.

\subsection{Sample patterning}

All DIC applications require the surface of the test sample to have a random, isotropic and high contrast surface pattern ('speckle pattern') as shown in figure 1. Pattern application methods used in macro-scale tests, such as airbrushing and using toner powder, are not suitable for the small-scale patterning needed for SEM-DIC. The speckles created with these methods allow for a minimum FOV of nominally $3 \times 3 \mathrm{~mm}$, assuming a toner particle size of $8 \mu \mathrm{m}$ and a $1024 \times 1024$ pixel imaging system. There have been a limited number of papers on the application of small-scale patterns suitable for SEM-DIC [4, 24-28]. Therefore, a full survey of patterning methods has been performed to determine the benefits and disadvantages of various patterning techniques.

\subsubsection{Chemical vapor thin film rearrangement. Chemical} vapor thin film rearrangement was investigated following a method first described by Scrivens et al in 2007 [26]. A $15 \mathrm{~nm}$ thick gold $\mathrm{Au}$ ) film was evaporated onto the surface of a polished $2024 \mathrm{Al}$ test sample using an Enerjet Evaporator at the University of Michigan's Lurie Nanofabrication Facility (LNF). The sample was then held in $95{ }^{\circ} \mathrm{C}$ water vapor for $9 \mathrm{~h}$. It had been previously observed by Scrivens et al that water vapor causes the Au film to break into a high-density dispersion of random nano-scale islands [26]. Although several attempts were made by the investigators to recreate this phenomenon using identical processing parameters as well as by varying parameters such as film thickness, vapor temperature, substrate material and sample surface roughness, repeatability was a significant issue. Small areas of the film broke up into islands as desired, but the islands were nonuniformly dispersed and the majority of the film remained intact. Because of the difficulties in obtaining repeatable patterns, this technique was not suitable for SEM-DIC and other methods were investigated.

2.2.2. Focused ion beam patterning. In 2007, Sabaté et al [35] used a FIB to mill a random pattern into the surface of a test sample for a DIC analysis. This technique was successful for Sabaté's work because a trench was milled through the 


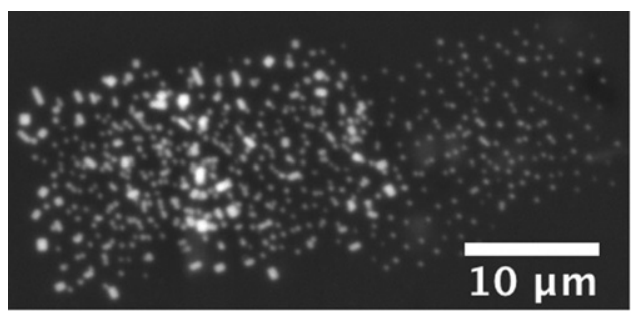

Figure 5. FIB pattern created by translation and rotation of a small user generated pattern. The substrate is 6061-T6 $\mathrm{Al}$ and the white spots are $\mathrm{Pt}$ that has been deposited on the surface with the ion beam. FIB patterning is expensive and can cause surface damage, but has potential for very small fields of view that need a high degree of accuracy.

patterned area to measure stress relief, and an EBSD map was not collected nor was the sample strained. However, the method is destructive and cannot be used in SEM-DIC tests coupled with EBSD, as the milled holes would create stress risers and interfere with crystallographic measurements. Instead of milling the surface, the authors instead deposited $\mathrm{Pt}$ markers onto the surface of a 6061-T6 Al test sample using a GIS equipped FIB in order to create a random tracking pattern. The approximately $20 \times 40 \mu \mathrm{m}$ pattern shown in figure 5 was created by first drawing an approximately $10 \times 10 \mu \mathrm{m}$ random block pattern in the FEI Quanta $2003 \mathrm{D}$ SEM FIB user interface and translating and rotating this block to tile the sample surface. A benefit of FIB patterning is its high repeatability and the ability to generate a desired pattern exactly as it is drawn in the user interface. One shortcoming of FIB patterning is the surface damage that results from $\mathrm{Ga}^{+}$ion implantation, which can be avoided by using the electron beam instead of the ion beam for deposition. However, using the electron beam in place of the ion beam increased processing time by a factor of 5 in the tungsten filament SEM used for these experiments. FIB patterning can also be expensive and is time consuming, taking nominally $20 \mathrm{~min}$ to generate a pattern in a $10 \times 10 \mu \mathrm{m}$ area. Additionally, FIB deposition is only capable of creating feature sizes down to $100 \mathrm{~nm}$ in width, which is only suitable for horizontal field widths down to nominally $35 \mu \mathrm{m}$. Generating a FIB pattern to cover a $35 \times 35 \mu \mathrm{m}$ FOV with the SEM would take approximately $4 \mathrm{~h}$. Although the FIB is not time efficient when a pattern is required over an entire $\mathrm{FOV}$, it has potential for tests requiring only a very small area of the FOV to be speckle patterned, such as when measuring stress relief around a milled trench. In these cases, FIB patterning would be capable of generating a suitable DIC pattern on a wide variety of conducting substrates.

2.2.3. Template patterning. Evaporating metal through TEM grids has been successfully used to apply a regular grid pattern to the surface of a test sample for deformation tracking [36-38]. In an effort to use a similar technique to generate a random pattern, alumina and polycarbonate filter membranes were used as templates for the evaporation of $\mathrm{Au}$ onto test sample surfaces. The alumina filters were $13 \mathrm{~mm}$ diameter, $65 \mu \mathrm{m}$ thick Whatman Anopore membrane filters with 100 and $200 \mathrm{~nm}$ diameter pores. The polycarbonate filters were 13 and $25 \mathrm{~mm}$ diameter, 6-10 $\mu \mathrm{m}$ thick SPI-Pore polycarbonate membrane filters with $0.1,0.4$ and $2 \mu \mathrm{m}$ diameter pores. First, a droplet of isopropyl alcohol (IPA) was applied to the surface of the test sample and the filter was placed on top of this drop. As the IPA evaporated, the membrane was pulled to the surface, creating an even contact between the membrane and the sample surface without any wrinkling or curling of the membrane edges. The membrane edges were then secured to the sample with $3 \mathrm{M}$ polyimide film tape. With the filter membrane securely attached to the sample surface, $5 \mathrm{~nm}$ of $\mathrm{Cr}$ followed by $500 \mathrm{~nm}$ of Au was evaporated onto the surface using an Enerjet Evaporator. Dome rotation in the evaporator was turned off to promote metal flow through the filter pores.

SEM imaging of the sample revealed that the alumina filter did not yield a pattern which can be attributed to the large filter thickness to pore diameter ratios of 325:1 (200 nm pore filter) and 650:1 (100 nm pore filter). The polycarbonate filters have a lower filter thickness to pore diameter ratio of 5:1 (2 $\mu \mathrm{m}$ pore filter), 25:1 (0.4 $\mu \mathrm{m}$ pore filter) and 60:1 (0.1 $\mu \mathrm{m}$ pore filter). SEM observations revealed that the metal was able to flow through only 0.4 and $2 \mu \mathrm{m}$ filters to the surface of the test sample as shown in figures $6(a)$ and $(b)$. The pattern created with these stock filters is not dense and would require numerous applications to achieve the necessary speckle density. The pattern was only observed where the filter membrane was in direct contact with the sample surface. Any gap between the filter and the substrate resulted in a hazy

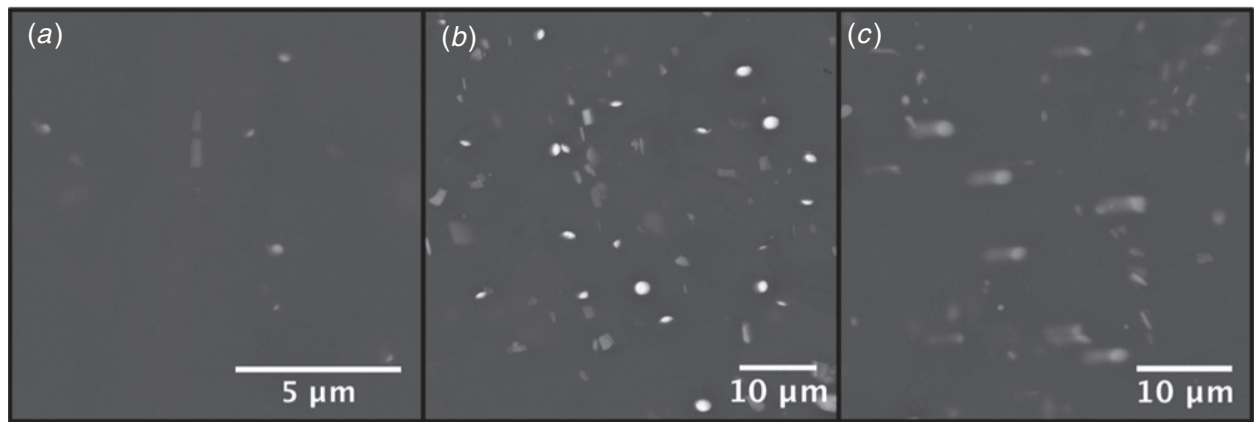

Figure 6. Speckles created by evaporating $5 \mathrm{~nm}$ of $\mathrm{Cr}$ followed by $500 \mathrm{~nm}$ of Au through polycarbonate membrane filters. A $0.4 \mu \mathrm{m}$ pore diameter filter was used for the speckles shown in $(a)$; a $2 \mu \mathrm{m}$ pore diameter filter was used for $(b)$. (c) The appearance of the speckles if the $2 \mu \mathrm{m}$ filter is not held tightly to the substrate surface during evaporation. 


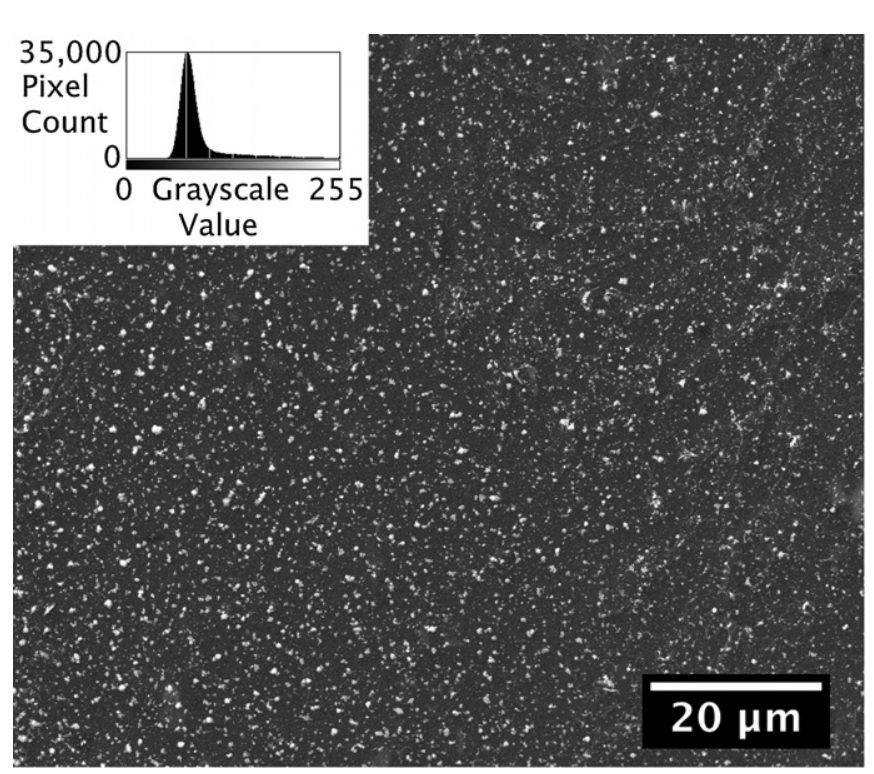

Figure 7. DIC appropriate $100 \mu \mathrm{m}$ HFW SEM image of a $100 \mathrm{~nm}$ diameter Au NP pattern on a polished 6061-T6 Al sample. The histogram in the top left shows the distribution of grayscale values over all of the image pixels. NP patterning is fast and inexpensive, but substrate dependent and lacks some repeatability in pattern location.

speckle or no speckle at all, as shown in figure 6(c). If the filter pore density can be improved, this method shows promise for larger FOV tests. This is an inexpensive patterning method, with polycarbonate filters costing approximately $\$ 0.65$ a piece.

2.2.4. Nanoparticle patterning. Au nanoparticles (NPs) are available in diameters of 2-250 $\mathrm{nm}$ and have good contrast when imaged under a SEM, making them promising patterning candidates for SEM-DIC experiments. In this work, Au NPs (diameters from 50 to $200 \mathrm{~nm}$ ) possessing a net negative charge and suspended in DI water were purchased from Ted Pella. The NPs were concentrated by allowing them to precipitate out of solution or by evaporating the solvent they were suspended in to concentrations up to $28 \times$ the stock concentration supplied by Ted Pella. They were then drop-cast or spincoated onto the sample surface to achieve a dense speckle pattern. NP patterning is a successful means of creating a suitable tracking pattern for SEM-DIC at HFWs ranging from 40 to $700 \mu \mathrm{m}$ (assuming $1024 \times 884$ pixel resolution). It is cost effective, costing only $\$ 56 / 20 \mathrm{~mL}$ from Ted Pella. NPs 10-20 nm in diameter can also be synthesized for under $\$ 1 / 20 \mathrm{~mL}$ following the Turkevich method [39]. This is a fast patterning process and yields a pattern in minutes. The resultant patterns contain a broad distribution of speckle sizes and exhibit high contrast when imaged with the secondary or backscatter electron detectors as shown in figure 7 . The biggest shortcomings with this method are the difficulty in preventing large agglomerates and in controlling pattern location.

The optimal method of NP solution concentration depends on the particle size. NPs $200 \mathrm{~nm}$ in diameter and greater naturally precipitate out of the solution in hours, while NPs near $100 \mathrm{~nm}$ in diameter can take days. Spinning the solution in a centrifuge to force the NPs to precipitate is another method that could potentially be investigated. After precipitation, a dropper or micropipette is used to remove DI water and further increase the concentration of the remaining solution. By using this method, $200 \mathrm{~nm}$ and $100 \mathrm{~nm}$ diameter NPs were concentrated to $28 \times\left(1.96 \times 10^{10} \mathrm{NP} \mathrm{mL}^{-1}\right)$ and $12 \times$ $\left(6.72 \times 10^{10} \mathrm{NP} \mathrm{mL}^{-1}\right)$ the supplied stock concentrations, respectively. NPs under $100 \mathrm{~nm}$ in diameter do not readily precipitate, and alternative concentration methods must be utilized. Placing the NP solution in a desiccant chamber successfully evaporated the solution, but unfortunately also concentrated any stabilizing agents or trace chemicals. These chemicals appeared as dendrite formations in the droplet, as shown in the SEM micrograph in figure $8(a)$. Rinsing the sample with DI water successfully removed the dendrites without removing NPs, as shown in figure $8(b)$. However, the effect of the presence of these concentrated chemicals on the clumping of the NPs has not yet been determined.

When the NP solution is allowed to sit undisturbed, NPs aggregate into large clumps that need to be broken up prior to application. To break up agglomerates, the NP solution was held in an ultrasonic cleaner for a minimum of $15 \mathrm{~min}$ immediately prior to drop casting. In addition to breaking up agglomerates, ultrasonic agitation also promoted a uniform particle dispersion in the solution. A micro-pipette or plastic dropper was used to drop cast the NP solution onto the sample surface.

Faster drying of the NP droplet greatly increases the quality of the speckle pattern, and numerous techniques (heating, angling the sample, spin-coating and layering) were investigated to decrease drying time. Long drying times provided the NPs with time to re-agglomerate, resulting in a non-homogeneous dispersion with the majority of NPs settling at the contact line of the evaporating droplet. This phenomenon is known as the 'coffee ring' effect. Ring formation is attributed to capillary fluid flow within the drying droplet carrying solute such as NPs to the contact line, which itself is pinned to the substrate. Higher concentrations of solute result in wider rings [40]. Heating was investigated as a means to increase pattern quality, wherein NPs were applied to a pure $\mathrm{Al}$ sample prior to heating the sample and NPs on a hotplate (model Corning PC-420D). However, heating the sample to temperatures that promote faster drying resulted in an increase in ring formation. Angling the sample as the NP solution dried assisted in controlling their final location upon evaporation, but also resulted in large agglomerates preferentially depositing at the lower-lying edge of the droplet. Spin-coating NPs onto the sample reduced the coffee ring effect; the spin-coated pattern was similar to the pattern observed in the center of a drop cast NP pattern but covered a larger area which is useful for wider FOV tests. Depositing carbon over a NP layer using an SPI Module Carbon Coater and a $2 \mathrm{~mm}$ diameter carbon fiber held the first layer in place and allowed a second NP droplet to better wet the surface of the sample, resulting in a thinner droplet and faster drying. However, the carbon layer breaks up following application of the second droplet, forming areas with a dense distribution of particles separated by regions of the substrate with a very diffuse covering of particles as shown 

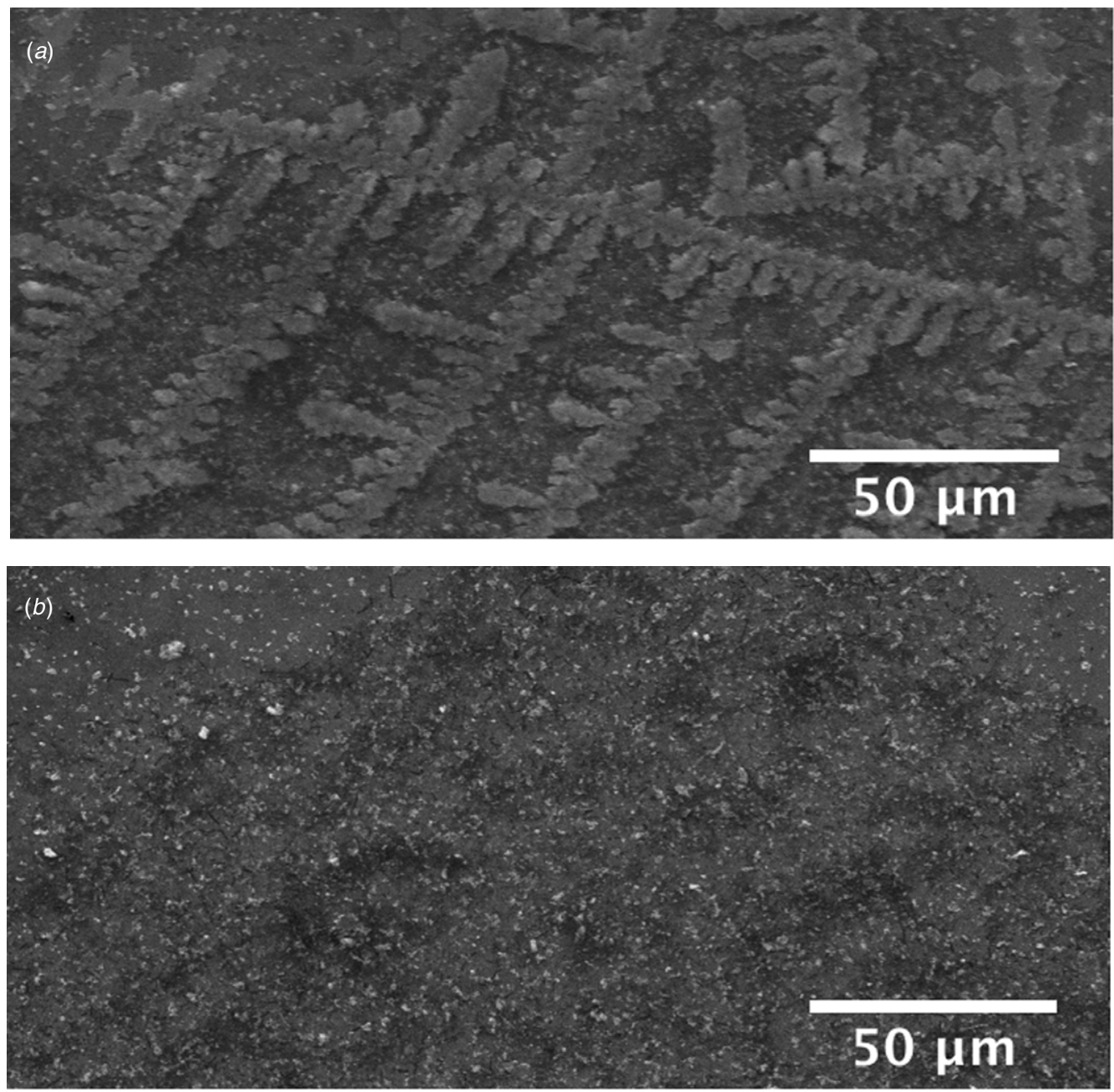

Figure 8. (a) Dendrites visible on the surface of a $99.99 \%$ pure Al test sample after applying concentrated $50 \mathrm{~nm}$ Au NPs. The dendrites resulted from the high concentration of a stabilizing agent remaining in solution. $(b)$ The same area of the sample after being rinsed in DI water to remove the dendrites.

in figure 9. While numerous techniques for NP patterning were investigated, the authors had the most success drop casting a small NP droplet onto the surface of the polished Al test sample and allowing it to air-dry while covered with a TEM grid mesh to prevent contamination from dust.

NP patterning is highly dependent on the substrate surface energy, with better patterns being achieved on substrates with a high surface energy. A droplet of NPs applied to a surface with high surface energy has a small contact angle and dries quickly relative to a droplet applied to a low surface energy surface, which will have a large contact angle. For pure Al and its alloys, a barrier oxide film with a thickness of approximately $1 \mathrm{~nm}$ forms immediately upon exposure of the polished surface to air [41]. The initial surface energy of this oxide film is high, and the NP droplet wets the surface in a thin layer that dries quickly from the outer edge to the center. Drying in this manner reduces the amount of clumping observed in the NPs and evenly distributes them in the center of the droplet resulting in a good DIC pattern. Over time, the oxide layer adsorbs polar water molecules from the atmosphere, which results in a drop in the surface energy (by as much as $75 \%$ for $\theta$-alumina) [42-44]. With the drop in surface energy, the contact angle between the $\mathrm{Al}$ substrate and the NP droplet increases, which results in longer drying times. Also, instead of drying from the edge of the drop to the center, the droplet now dries to a thin layer before the meniscus breaks, at which point the remaining fluid dries at the edge of the original droplet. Following this drying scheme, a large ring of NPs accumulate at the edge of the droplet and few NPs are observed in the droplet center. It is therefore suggested that all test samples be stored with desiccant to slow the adsorption of water at the oxide surface and prevent heterogeneous dispersion. On $\mathrm{Al}$ samples with low surface energy, it is possible to use the tip of a plastic dropper or micropipette to drag the edges of the droplet out and decrease the contact angle. This encourages the droplet to dry in a method similar to that seen on samples with high surface energies.

NP patterning is a fast and inexpensive way to apply a displacement-tracking pattern for SEM-DIC. Pattern density can easily be controlled by concentration of the NP solution. While some clumping is desired to create a random pattern, care must be taken to break up large agglomerates with an ultrasonic cleaner before application. To yield the best pattern, drying time should be reduced by using a small NP droplet 


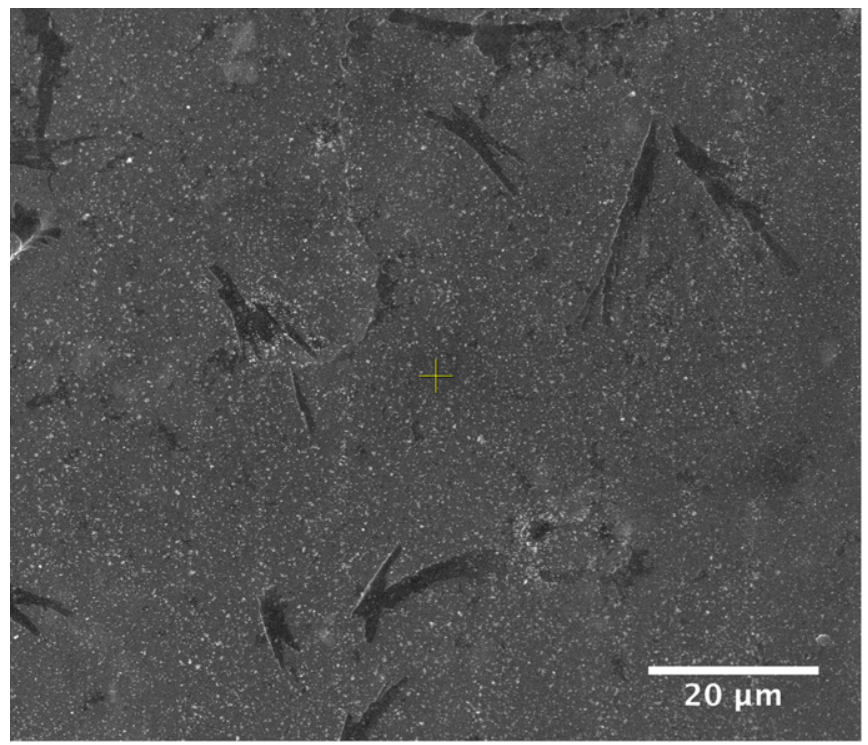

Figure 9. Two drop cast layers of NPs applied to a 99.99\% Al sample. A carbon layer sputtered from a $2 \mathrm{~mm}$ diameter carbon fiber was applied between NP layers to hold the first layer in place and speed drying of the top layer. The dark lines are cracks in the carbon layer that occurred after the second NP droplet was applied.

and if using Al, applying the NP solution to a freshly polished surface.

\subsubsection{EBSD-assisted nanoparticle patterning. In SEM-DIC} experiments combined with EBSD, a surface pattern is often desired directly over the EBSD area. In this work, $200 \mathrm{~nm}$ $\mathrm{Au}$ NPs applied to the sample surface following an EBSD analysis display a preferred arrangement within the EBSD scan area. This preferred arrangement has been observed on $99.99 \%$ pure $\mathrm{Al}$ and $6061-\mathrm{T} 6 \mathrm{Al}$. The surface of a $99.99 \%$ pure Al test sample following EBSD with a beam voltage of $25 \mathrm{kV}$, an aperture of 3, and a spot size of 6 is shown in figure $10(a)$. A hexagonal grid of dark spots representing the EBSD scan locations is visible on the surface. Immediately after removal of the test sample from the SEM, $200 \mathrm{~nm}$ diameter Au NPs

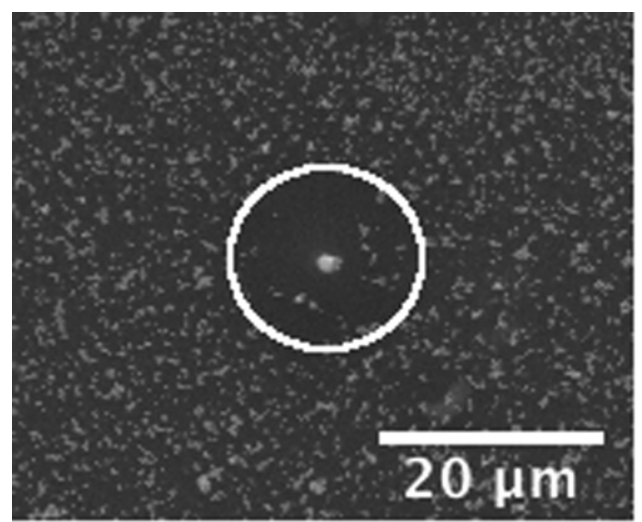

Figure 11. Image of an $\mathrm{Al}$ substrate with a $1 \mu \mathrm{m}$ diameter FIB-deposited Pt marker at the center. While the majority of the surface is covered by $200 \mathrm{~nm}$ diameter Au NPs, the area around the Pt marker is free of NPs. The size of the NP free area scales with the marker size.

were applied over the EBSD area. Figure 10(b) shows the surface of the sample following the application of the NPs, with a random arrangement of NP agglomerates visible in the area where no EBSD was performed. In the EBSD affected area, the NPs collected into pools following the grid of EBSD collection points, shown by the ovals in figure $10(b)$. The preferred pooling of NPs could be exploited to produce patterns in the exact area that the EBSD analysis was performed. Similar results were not seen with $100 \mathrm{~nm}$ NPs. Atomic force microscopy (AFM) was used to study the topography of the area displaying the grid pattern. The AFM did not reveal any topographical changes when points of the grid were crossed; therefore, the pooling of NPs cannot be explained by changes in surface elevation.

In addition to the tendency of NPs to order themselves in an EBSD-analyzed area, it has been observed that NPs do not adhere to an Al surface near the Pt FIB markers, as shown in figure 11. The repulsion of NPs in the area around the Pt markers occurs regardless of whether the markers are deposited with an electron or ion beam. The area free of NPs can be reduced by reducing the Pt marker size. The area free of NPs extends beyond the Pt halo that surrounds the


Figure 10. (a) Periodic surface grid pattern visible on a 99.99\% pure Al test sample post EBSD. A $25 \mathrm{kV}$ accelerating voltage and spot size of 6 was used for the EBSD analysis. (b) $200 \mathrm{~nm}$ diameter Au NPs preferentially depositing in the grid area. The area below the line was analyzed by EBSD and shows a preferred arrangement of NP agglomerates while the area above the line was not analyzed by EBSD and shows a random distribution of NP agglomerates. 


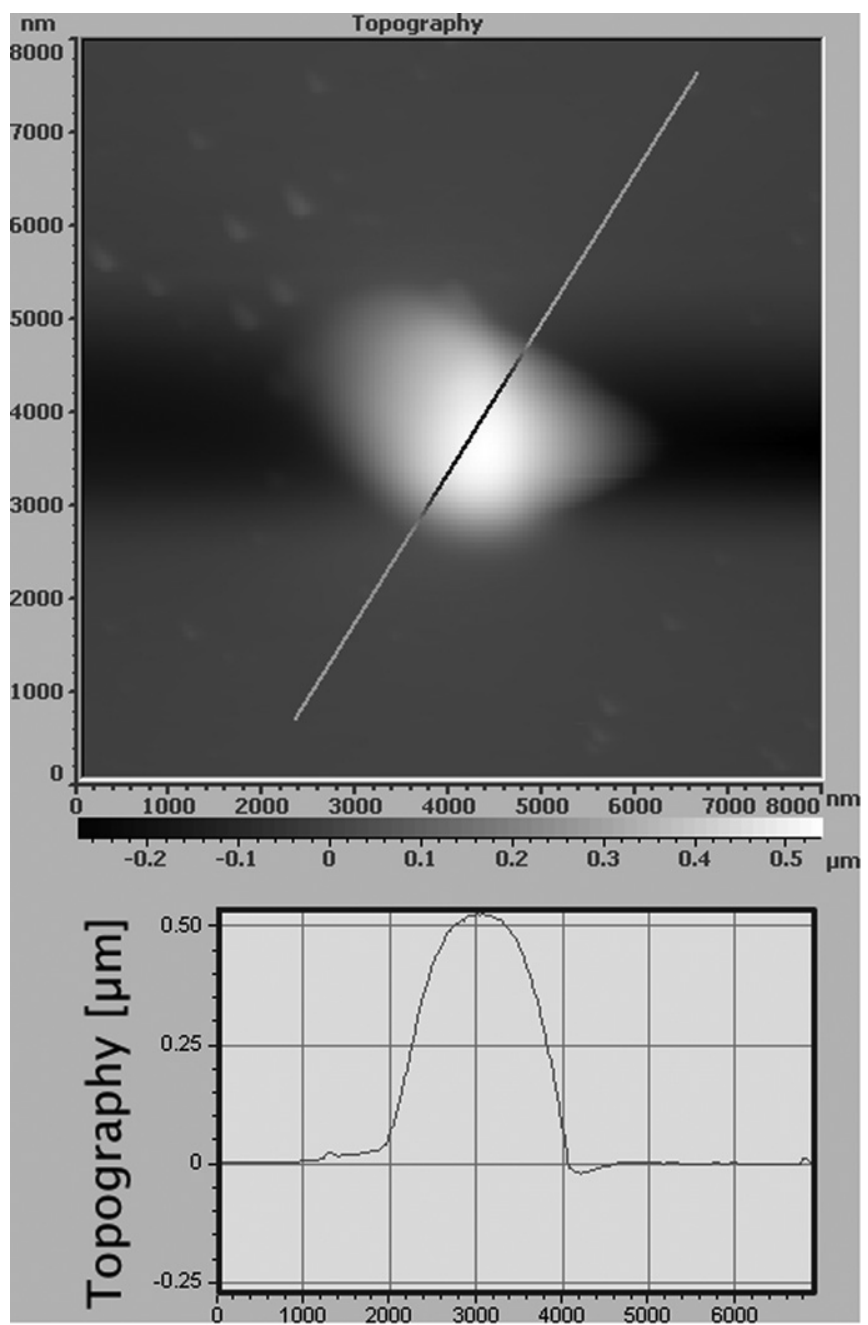

Figure 12. AFM topographical map of a $1 \mu \mathrm{m}$ diameter Pt marker. The profile along the diagonal line is shown in the lower image. The surface remains flat in the vicinity of the Pt marker, suggesting that it is not a change in elevation that prevents NPs from adhering to the surface around the marker.

marker. This phenomenon was investigated by AFM, and no topographical changes were observed on the surface as the $\mathrm{Pt}$ marker was approached, as shown in figure 12.

Nanoparticle pattern location can be precisely controlled on Al by drop casting $200 \mathrm{~nm}$ Au NPs onto the sample surface following pre-test EBSD mapping. By changing the EBSD grid spacing, the speckle size and spacing between speckles can be adjusted. This preferential arrangement has been successfully used for $300 \times 260 \mu \mathrm{m}$ and $700 \times 600 \mu \mathrm{m}$ FOV tests. When using this and any other NP patterning technique, care must be taken if FIB-deposited Pt markers are used for FOV alignment, as NPs do not adhere to the sample surface near the markers.

2.2.6. Electron beam lithography. Surface patterning using electron beam (e-beam) lithography patterning was performed with a Raith-150 e-beam system. E-beam lithography is a well-known process for prototyping integrated circuits and photo-mask creation as well as research into the effect of scaling on integrated circuits and physical phenomena at

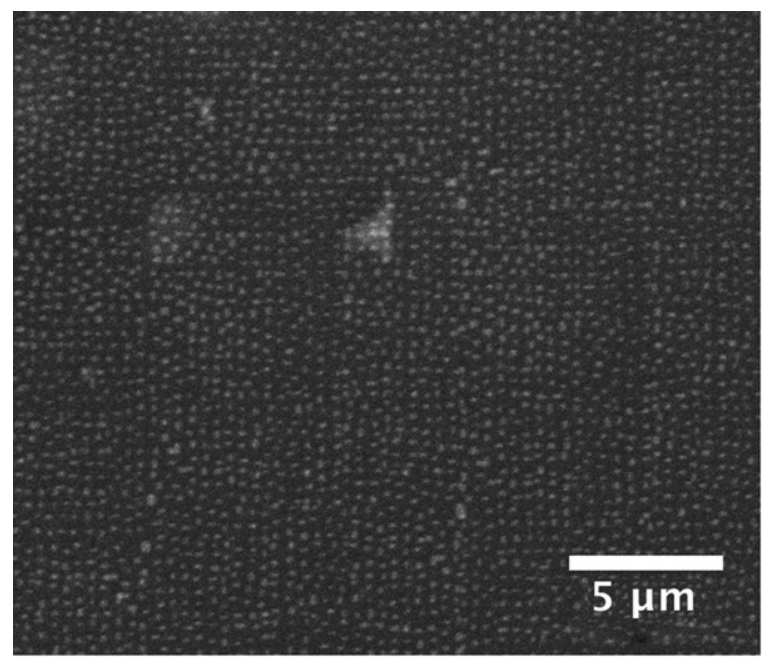

Figure 13. A section of an e-beam lithography pattern. The substrate is 6061-T6 Al and the speckles are $\mathrm{Au}$ with a $\mathrm{Cr}$ adhesion layer that has been evaporated onto the surface. The pattern consists of rectangles with varying heights and widths.

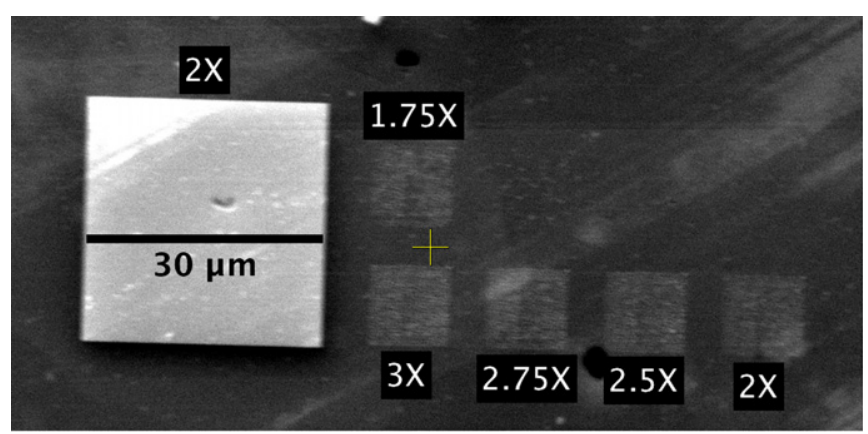

Figure 14. $10 \mu \mathrm{m} \mathrm{Au}$ speckle patterns on a 6061-T6 Al substrate exposed at several e-beam dose levels (base dose was $100 \mu \mathrm{C} \mathrm{cm}^{-2}$ ) can be seen on the right side of the image. Doses of $1.5 \times, 1.25 \times$ and $1 \times$ the base dose were also used but did not fully expose. A $30 \mu \mathrm{m}$ marker (left) is used to locate the pattern during SEM imaging.

the nano-scale. For detailed information about the e-beam process, one can refer to a wide array of literatures on its development and use (for example, see [45, 46] and the references contained therein). To use e-beam lithography to create a small-scale DIC pattern, a surface speckle pattern was first drawn in the Raith software's computer aided design (CAD) editor (Version 3.0 SP 9). This pattern contained rectangles of varying aspect ratios distributed in a random and isotropic manner, as shown in figure 13. The pattern can be readily scaled up or down to accommodate DIC at various magnifications. A large marking feature, shown in figure 14, was drawn next to the speckle pattern to assist in locating the patterned area during SEM imaging.

An image of the speckle pattern was exposed into a thin layer of polymethyl methacrylate (MicroChem 950 PMMA A2) which was spin-coated on the substrate surface at $2000 \mathrm{rpm}$ for $30 \mathrm{~s}$. During the exposure process, the PMMA at the speckle pattern locations came into direct contact with the e-beam and was broken up into fragments that could be easily dissolved by developer. Following exposure, the PMMA was developed in a solution of methyl isobutyl ketone (MIBK) and 
Table 1. Summary of patterning techniques for SEM-DIC.

\begin{tabular}{|c|c|c|}
\hline Technique & Advantages & Disadvantages \\
\hline Focused ion beam patterning & $\begin{array}{l}\text { Accurate control of pattern location } \\
\text { Repeatable } \\
\text { Substrate independent }\end{array}$ & $\begin{array}{l}\text { Expensive } \\
\text { Time consuming } \\
\text { Ion beam surface damage }\end{array}$ \\
\hline Template patterning & $\begin{array}{l}\text { Fast } \\
\text { Inexpensive } \\
\text { Numerous samples can be patterned } \\
\quad \text { at once } \\
\text { Repeatable } \\
\text { Accurate control of pattern location } \\
\text { Substrate independent }\end{array}$ & $\begin{array}{l}\text { Filter must be held perfectly flat to } \\
\text { the surface of the sample } \\
\text { Stock filter pore aspect ratio limits } \\
\text { the minimum feature size to } 400 \mathrm{~nm} \\
\text { Low pore density in stock filters } \\
\text { requires numerous applications }\end{array}$ \\
\hline Nanoparticle patterning & $\begin{array}{l}\text { Fast } \\
\text { Inexpensive } \\
\text { Can be stock purchased with } \\
\quad \text { diameters ranging from } 2 \text { to } 250 \mathrm{~nm} \\
\text { Easily synthesized } \\
\text { Broad distribution of speckle sizes }\end{array}$ & $\begin{array}{l}\text { Difficult to control pattern location } \\
\text { Difficult to achieve repeatable results } \\
\text { Substrate dependent }\end{array}$ \\
\hline E-beam lithography & $\begin{array}{l}\text { Accurate control of pattern location } \\
\text { Repeatable } \\
\text { Easily scaled for different } \\
\quad \text { magnifications } \\
\text { Suitable for high temperature } \\
\text { Substrate independent }\end{array}$ & $\begin{array}{l}\text { Expensive } \\
\text { Time consuming } \\
\text { Multi-step process }\end{array}$ \\
\hline
\end{tabular}

isopropyl alcohol (proportions of 1:3 by volume respectively). The developing process dissolved the exposed areas of PMMA, leaving behind a bare substrate. A $5 \mathrm{~nm}$ adhesion layer of $\mathrm{Cr}$ was then evaporated onto the sample, followed by $25 \mathrm{~nm}$ of $\mathrm{Au}$. $\mathrm{Cr}$ bonds well with oxygen and remains metallic in the vacuum chamber. Thus $\mathrm{Au}$, which forms strong bonds with metals but weak bonds with oxygen, adheres better on oxidized or hydroxide substrate surfaces following a $\mathrm{Cr}$ adhesion layer $[47,48]$. The PMMA film was removed by holding the sample in acetone, leaving behind only the $\mathrm{Cr} / \mathrm{Au}$ layer that was deposited on the sample surface. The sample was then placed in an ultrasonic cleaner for $12 \mathrm{~min}$ to expedite the lift-off process and validate pattern adhesion.

Successful exposure of a pattern using e-beam lithography requires establishing an accurate measurement of the working distance between the e-beam and substrate in the patterning region. A change in the surface height between the focusing feature and the pattern area as small as $10 \mu \mathrm{m}$ has resulted in a failed exposure. To do this, a feature(s) used for focusing should be located as close to the desired pattern area as possible. The authors used nickel nano-beads applied with a small foam-tipped applicator, or FIB deposited Pt markers, as focusing features. As added security, multiple focusing features surrounding the desired pattern area were used so that an average working distance could be calculated for pattern exposure.

Determination of the appropriate e-beam dose is also critical, and a dose test should be performed before attempting to expose a speckle pattern for DIC. The thickness of the PMMA layer determines the dose of the e-beam. Thicker layers require a higher e-beam dose to expose the PMMA layer. If the layer is too thin, material evaporated onto the developed PMMA may form a continuous film instead of discrete disconnects at the speckle pattern features. Here, a film thickness of $100 \mathrm{~nm}$ allows for a dose of $275 \mu \mathrm{C} \mathrm{cm}^{-2}$ at $20 \mathrm{kV}$ and ensured that a $25 \mathrm{~nm}$ Au layer evaporated onto the exposed area is discontinuous at the pattern features.

E-beam lithography is capable of generating consistently high quality patterns for FOVs as small as $10 \times 10 \mu \mathrm{m}$, a feat that has not yet been achieved with NPs. Patterns larger than the $100 \mu \mathrm{m}$ writefield of the Raith 150 e-beam can be generated by stitching pattern areas together in multiple exposures. E-beam lithography is also capable of generating patterns appropriate for high temperature tests, by evaporating a high temperature material like alumina onto the substrate in place of $\mathrm{Au}$. The disadvantages of e-beam lithography are its high cost and its limited applicability to non-flat substrates due to the method's sensitivity to working distance. Additionally, while the time in the e-beam system may be as short as an hour, the time from spin coating the resist to lifting it off to reveal the pattern can take over a day.

\section{Summary}

FIB, template, nanoparticle and e-beam lithography patterning of samples for SEM-DIC were investigated. All the techniques have successfully been applied to Al test samples, and have their relative advantages and disadvantages. Choosing the correct technique requires careful consideration of test factors, including FOV, substrate characteristics, cost, location dependence and repeatability. FIB patterning can produce a pattern with speckle diameters down to $100 \mathrm{~nm}$. However, it is a time-consuming technique and is not an efficient way to pattern a large area. FIB patterning has potential in tests where a highly controlled pattern is only required in a small area of 
the FOV. Template patterning through stock polycarbonate membrane filters can produce patterns with $0.4 \mu \mathrm{m}$ speckle diameters and is a quick process, requiring only the time to pump down the evaporator/sputter-coater chamber and deposit the metal film. However, the filter pores are not dense, and evaporation through multiple filters would be required. Nanoparticle (NP) patterning has been used successfully to generate patterns with speckle diameters down to $10 \mathrm{~nm}$. The greatest shortcoming of NP patterning is controlling the location of the area with a suitable DIC-quality pattern. The preferential arrangement of $200 \mathrm{~nm}$ diameter Au NPs on an Al surface following EBSD can be used for tests with FOVs of approximately $300 \times 300 \mu \mathrm{m}$. E-beam lithography has the greatest repeatability of any of the analyzed methods. An ebeam SEM-DIC pattern can easily be scaled up or down, was successfully applied to both ceramic and Al substrates, and can be used to create a pattern for high temperature testing. The greatest shortcoming of e-beam patterning is the cost related to the e-beam usage. A summary of patterning methodologies for SEM-DIC is shown in table 1.

\section{Acknowledgments}

The authors gratefully acknowledge the financial support of the National Science Foundation, contract no 0927530, monitored by Glaucio Paulino. The authors thank the US Department of Energy, Office of Basic Energy Sciences, contract no DESC0003996, monitored by Dr John Vetrano, for supporting the development of the DIC-SEM distortion correction codes used in this work. Support for this work was also provided by the University of Michigan's Rackham Graduate School Non-Traditional Student Fellowship. The authors would like to acknowledge Jared Tracy for assistance in investigating patterning methods and Dr John Mansfield for SEM training and advice. The authors thank Professor Pramod Reddy and Aaron Tan for the use of their AFM and their experimental assistance. Portions of this work were performed at the Lurie Nanofabrication Facility, a member of the National Nanotechnology Infrastructure Network, which is supported in part by the National Science Foundation. This work was also performed in the University of Michigan's Electron Microbeam Analysis Laboratory.

\section{References}

[1] Sutton M A, Li N, Garcia D, Cornille N, Orteu J J, McNeill S R, Schreier H W and Li X 2006 Metrology in a scanning electron microscope: theoretical developments and experimental validation Meas. Sci. Technol. 17 2613-22

[2] Sutton M A, Li N, Joy D C, Reynolds A P and Li X 2007 Scanning electron microscopy for quantitative small and large deformation measurements: part I. SEM imaging at magnifications from 200 to 10,000 Exp. Mech. 47 775-87

[3] Sutton M A, Li N, Joy D C, Reynolds A P and Li X 2007 Scanning electron microscopy for quantitative small and large deformation measurements: part II. Experimental validation for magnifications from 200 to 10,000 Exp. Mech. 47 789-804
[4] Tschopp M A, Bartha B B, Porter W J, Murray P T and Fairchild S B 2009 Microstructure-dependent local strain behavior in polycrystals through in situ scanning electron microscope tensile experiments Metall. Mater. Trans A $402363-8$

[5] Kang J, Ososkov Y, Embury J D and Wilkinson D S 2007 Digital image correlation studies for microscopic strain distribution and damage in dual phase steels Scr. Mater. 56 999-1002

[6] Sutton M A, Orteu J J and Schreier H W 2009 Image Correlation for Shape, Motion and Deformation Measurements, Basic Concepts, Theory and Applications (New York: Springer)

[7] Daly S H 2010 Digital image correlation in experimental mechanics for aerospace materials and structures Encyclopedia of Aerospace Engineering ed R Blockley and W Shyy (New York: Wiley)

[8] Peters W H and RansonW F 1982 Digital imaging techniques in experimental stress analysis Opt. Eng. 21 427-31

[9] Sutton M A, Wolters W J, Peters W H, Ranson W F and McNeill S R 1983 Determination of displacements using an improved digital correlation method Image Vision Comput. 1 133-9

[10] Pierron F, Sutton M A and Tiwari V 2011 Ultra high speed DIC and virtual fields method analysis of a three point bending impact test on an aluminium bar Exp. Mech. $51537-63$

[11] Kirugulige M S and Tippur H V 2009 Measurement of fracture parameters for a mixed-mode crack driven by stress waves using image correlation technique and high speed digital photography Strain 45 108-22

[12] Gilat A, Schmidt T E and Walker A L 2009 Full field strain measurement in compression and tensile split Hopkinson bar experiments Exp. Mech. 49 291-302

[13] Parsons E M, Weerasooriya T, Sarva S and Socrate S 2010 Impact of woven fabric: experiments and mesostructure-based continuum-level simulations J. Mech. Phys. Solids 58 1995-2021

[14] Kim K and Daly S 2011 Martensite strain memory in the shape memory alloy nickel-titanium under mechanical cycling Exp. Mech. 51 641-52

[15] Renard K, Ryelandt S and Jacques P J 2010 Characterization of the portevin-Le Châtelier effect affecting an austenitic TWIP steel based on digital image correlation Mater. Sci. Eng. A 527 2969-77

[16] Nicoletto G, Anzelotti G and Riva E 2009 Mesoscopic strain fields in woven composites: experiments vs. finite element modeling Opt. Laser Eng. 47 352-9

[17] Lai J H and Levenston M E 2010 Meniscus and cartilage exhibit distinct intra-tissue strain distributions under unconfined compression Osteoarthr. Cartilage 18 1291-9

[18] Efstathiou C, Sehitoglu H and Lambros J 2010 Multiscale strain measurements of plastically deforming polycrystalline titanium: role of deformation heterogeneities Int. J. Plast. 26 93-106

[19] Hamilton R F, Dilibal S, Sehitoglu H and Maier H J 2011 Underlying mechanism of dual hysteresis in NiMnGa single crystals Mater. Sci. Eng. A 528 1877-81

[20] Jonnalagadda K N, Chasiotis I, Yagnamurthy S, Lambros J, Pulskamp J, Polcawich R and Dubey M 2010 Experimental investigation of strain rate dependence of nanocrystalline $\mathrm{Pt}$ films Exp. Mech. 50 25-35

[21] Yoneyama S, Kitagawa A, Iwata S, Tani K and Kikuta H 2007 Bridge deflection measurement using digital image correlation Exp. Tech. 31 34-40

[22] Tatschl A and Kolednik O 2003 A new tool for the experimental characterization of micro-plasticity Mater. Sci. Eng. A 339 265-80 
[23] Tanaka Y, Naito K, Kishimoto S and Kagawa Y 2011 Development of a pattern to measure multiscale deformation and strain distribution via in situ FE-SEM observations Nanotechnology 22115704

[24] Sutton M A, McNeill S R, Helm J D and Chao Y J 2000 Advances in two-dimensional and three-dimensional computer vision photomechanics Top. Appl. Phys. 77 323-72

[25] Berfield T A, Patel J K, Shimmin R G, Braun P V, Lambros J and Sottos N R 2007 Micro- and nanoscale deformation measurement of surface and internal planes via digital image correlation Exp. Mech. 47 51-62

[26] Scrivens W A, Luo Y, Sutton M A, Collete S A, Myrick M L, Miney P, Colavira P E, Reynolds A P and Li X 2007 Development of patterns for digital image correlation measurements at reduced length scales Exp. Mech. 47 63-77

[27] Collette S A et al 2004 Development of patterns for nanoscale strain measurements: I. fabrication of imprinted Au webs for polymeric materials Nanotechnology 15 1812-7

[28] Li N, Guo S and Sutton M A 2011 Recent progress in e-beam lithography for SEM patterning MEMS and Nano., Volume 2, Conf. Proc. Soc. for Exp. Mech. Ser. 2, ed T Proulx (New York: Springer) pp 163-6

[29] Carroll J, Abuzaid W, Lambros J and Sehitoglu H 2010 An experimental methodology to relate local strain to microstructural texture Rev. Sci. Instrum. 81083703

[30] Zhu T, Sutton M A, Li N, Orteu J J, Cornille N, Li X and Reynolds A P 2011 Quantitative stereovision in a scanning electron microscope Exp. Mech. 51 97-109

[31] Schwartz A J, Kumar M, Adams B L and Field D P (ed) 2009 Electron Backscatter Diffraction in Materials Science (New York: Springer)

[32] Maitland T and Sitzman S 2007 Scanning Microscopy for Nanotechnology, Techniques and Applications ed W Zhou and Z L Wang (New York: Springer) pp 41-75

[33] Reyntjens S and Puers R 2001 A review of focused ion beam applications in microsystem technology J. Micromech. Microeng. 11 287-300

[34] Conversation with FEI Service Engineer, 8 April 2011

[35] Sabaté N, Vogel D, Keller J, Gollhardt A, Marcos J, Gràcia I, Cané C and Michel B 2007 FIB-based technique for stress characterization on thin films for reliability purposes Microelectron. Eng. 84 1783-7

[36] Biery N, DeGraef M and Pollock T M 2003 A method for measuring microstructural-scale strains using a scanning electron microscope: applications to $\gamma$-titanium aluminides Metall. Mater. Trans. A 34 2301-13

[37] Biery N E, DeGraef M and Pollock T M 2001 Influence of microstructure and strain distribution on failure properties in intermetallic TiAl-based alloys Mater. Sci. Eng. A 319-321 613-7

[38] Castelluccio G M, Yawny A A, Perez Ipiña J E and Ernst H A 2011 In situ evaluation of tensile properties of heat-affected zones from welded steel pipes Strain online, doi:10.1111/j.1475-1305.2010.00799.x

[39] Turkevich J, Stevenson P C and Hillier J 1951 A study of the nucleation and growth processes in the synthesis of colloidal gold Discuss. Faraday Soc. 11 55-75

[40] Deegan R D, Bakajin O, Dupont T F, Huber G, Nagel S R and Witten T A 1997 Capillary flow as the cause of ring stains from dried liquid drops Nature 389 827-9

[41] Davis J R (ed) 1993 Aluminum and Aluminum Alloys (Materials Park, OH: ASM International) p 579

[42] Yan B D, Meilink S L, Warren G W and Wynblatt P 1987 Water adsorption and surface conductivity measurements on $\alpha$-alumina substrates IEEE Trans. Comps. Hybrids Manuf. Tech. 10 247-51

[43] Sasaki S and Pethic J B 2000 Effects of surrounding atmosphere on micro-hardness and tribological properties of sintered alumina Wear $241204-8$

[44] Łodziana Z, Topsøe N Y and Nørskov J K 2004 A negative surface energy for alumina Nat. Mater. 3 289-93

[45] McCord M A and Rooks M J 1997 Handbook of Microlithography, Micromachining, and Microfabrication vol 1, ed P Ray-Choudhury (Bellingham, WA: SPIE/London: IEE) pp 139-250

[46] Londe G, Han A and Cho H J 2007 Functional Nanostructures: Processing, Characterization, and Applications ed S Seal (New York: Springer) pp 119-24

[47] Franssila S (ed) 2010 Introduction to Microfabrication (Chichester, UK: Wiley) p 216

[48] Köhler M and Fritzsche W (ed) 2007 Nanotechnology: An Introduction to Nanostructuring Techniques (Weinheim: Wiley-VCH) p 38 